Sharif University of Technology
Scientia Iranica
Transactions B: Mechanical Engineering
heIENTIA

Research Note

\title{
Study of swelling behavior of temperature-sensitive hydrogels considering inextensibility of network
}

\author{
H. Mazaheri* \\ Department of Mechanical Engineering, Bu-Ali Sina University, Hamedan, Iran.
}

Received 28 September 2017; received in revised form 30 November 2017; accepted 30 January 2018

\section{KEYWORDS}

Temperature sensitive hydrogels;

Homogeneous and

inhomogeneous

swelling;

Chain locking;

Finite-element

method.

\begin{abstract}
In this work, the equilibrium swelling of temperature-sensitive hydrogel networks is studied with an emphasis on the chain locking of the network. Using the Gent model for an elastic part of free energy and modifying the mixing part of the free energy, a continuous model is developed which considers inextensibility of the network chains and has good agreement with the experimental data, particularly for smaller values of cross-linking density of the network and larger values of the swelling ratio. Following the validation of the modified model, it is employed to study the inhomogeneous swelling of a spherical shell on a hard core both analytically and numerically. The analytical solution is used to validate the numerical method. Finally, the inhomogeneous swelling of a bilayer beam with an active temperature sensitive hydrogel is investigated, and the results are presented. The obtained results show the importance level of considering the chain locking in swelling of the network in the applied problems.
\end{abstract}

(C) 2019 Sharif University of Technology. All rights reserved.

\section{Introduction}

Smart hydrogels composed of covalently cross-linked polymeric network that can respond to environmental stimuli such as temperature [1-3], pH [4-6], mechanical load $[7,8]$, ionic and salt concentration $[9,10]$, light $[11-$ $13]$, and electric field $[14,15]$ and can imbibe large amount of water and experience a large deformation. These materials can be used as a sensor and an actuator simultaneously. Thus, they have a promising future for application in drug delivery systems [16], sensors and actuators [17-21], and microfluidics and microvalves [22-24].

Temperature-sensitive hydrogels represent a class of smart hydrogels that attracted a great deal of interest in the literature, especially in the field of constitutive modelling. To describe the behavior of

\footnotetext{
*. E-mail address: h.mazaheri@basu.ac.ir
}

these materials, a coupled thermo-mechanical theory employed for precise prediction of their behavior in complicated problems appears in the applications. Thus, coupled thermo-mechanical behavior of the temperature-sensitive hydrogels has been a subject of interest, especially in recent years [25-28].

Considering a sharp front separating swelling and collapsed phases, Ji et al. [29] developed a constitutive model in the scope of the extended finiteelement method for thermally induced volume transitions in stimulus-responsive hydrogels. Birgersson et al. [27] derived a model for transient behavior of a temperature-sensitive hydrogel by employing a biphasic approach. Chester and Anand [25] developed a model for the thermally responsive elastomeric gels accounting for large deformation and coupled thermomechanical conditions. They considered inextensibility of the polymer chains by employing a non-Gaussian model for the change in configurational entropy of the polymer chains. Cai and Suo [26] developed a constitutive model for the temperature-sensitive poly- 
(N-isopropylacrylamide) hydrogel (PNIPAM hydrogel) which has good agreement with the experimental data. However, in the vicinity of the Phase-Transition Temperature (PTT), multiple-solution and snap-through instability was observed resulting in limitations to using the model in finite-element implementations and also in inhomogeneous swelling problems. This problem was solved by Mazaheri et al. [28] by assuming a modified definition for the mixing free energy by expanding the logarithmic term in the mixing free energy considered in the work of Cai and Suo [26].

In this work, polymer chain inextensibility is considered using the Gent model for elastic part of the free energy density. By means of the Gent model, the locking parameter added to calculations can be used as an extra curve-fitting parameter to predict the experimental data precisely. Moreover, parallel with the work of Mazaheri et al. [28], the mixing part of the free energy is modified to attain a continuous constitutive model for PNIPAM hydrogels which can eliminate the multiple-solution instability problems. In addition, the effect of the inextensibility on the deformation of PNIPAM hydrogels is studied due to temperature changes by solving some analytical and numerical examples.

The paper is organized as follows. Firstly, the model is illustrated in Section 2. Thereafter, the validity and stability of the model in the vicinity of PTT are investigated in Section 3 by solving homogeneous swelling problems (Subsection 3.2) and inhomogeneous swelling ones (Subsection 3.2). Finally, summary and conclusions are presented in Section 4.

\section{Model description}

Consider an element of a hydrogel in reference (dry) and the current states in which the element's coordinates are denoted by $\mathbf{X}$ and $\mathbf{x}(\mathbf{X})$, respectively. Based on this definition, deformation gradient $\mathbf{F}$ and right Cauchy-Green deformation tensor $\mathbf{C}$ can be defined as follows:

$$
\begin{aligned}
& \mathbf{F}=\frac{\partial \mathbf{x}}{\partial \mathbf{X}}, \\
& \mathbf{C}=\mathbf{F}^{T} \mathbf{F} .
\end{aligned}
$$

Helmholtz free energy density of an arbitrary element of the hydrogel per unit reference volume is considered here. In this work, the equilibrium swelling of the hydrogel is studied. Thus, by neglecting the timedependent diffusion phenomenon and employing a variational approach to this condition, the change in the Helmholtz free energy is expressed as follows [26,28]:

$$
\delta W=\mathbf{P}: \delta \mathbf{F}+\mu \delta c,
$$

where $\mathbf{P}, \mu$, and $c$ are the first Piola-Kirchhoff stress tensor, chemical potential, and number of fluid molecules absorbed by the network per unit volume of the dry polymer, respectively. The Helmholtz free energy density function is a function of deformation, number of solvents, and absolute temperature, $T$, and its variations can be calculated in terms of these variables. Considering the free energy as $W=W(\mathbf{F}, c, T)$ and substituting it in Eq. (3) yields:

$$
\mathbf{P}=\frac{\partial}{\partial \mathbf{F}} W(\mathbf{F}, c, T), \quad \mu=\frac{\partial}{\partial c} W(\mathbf{F}, c, T) .
$$

To define the free energy functionality, an additive decomposition of the free energy density is assumed in the form of (e.g., [7,25,26,30-32] among others):

$$
W=W_{\text {stretch }}(\mathbf{F}, T)+W_{\text {mixing }}(c, T),
$$

where $W_{\text {stretch }}(\mathbf{F}, T)$ and $W_{\text {mixing }}(c, T)$ are elastic and mixing parts of the free energy density, respectively [33]. The network is assumed incompressible; as a result, the change in the hydrogel volume originates only from the fluid molecules diffusion through the network. This constraint is expressed as below [30]:

$$
J=1+\nu c,
$$

where $\nu$ and $J$ are volume of a fluid molecule and the determinant of $\mathbf{F}$, respectively. This constraint is considered using Lagrange multiplier method and modifying the free energy function as follows $[9,30]$ :

$$
W=W_{\text {stretch }}(\mathbf{F}, T)+W_{\text {mixing }}(c, T)+\Pi(J-1-\nu c),
$$

where $\Pi$ is the Lagrange multiplier that should be defined from boundary conditions. The constitutive equations of Eq. (4) should be reformed due to modification of the free energy. Rewriting the constitutive laws and eliminating the Lagrange multiplier yields the stress function. Considering pure water as a solvent with respect to the equilibrium state, the chemical potential is constant and equal to zero, and, in this way, the stress tensor can be obtained as follows:

$$
\mathbf{P}=\frac{\partial}{\partial \mathbf{F}} W_{\text {stretch }}(\mathbf{F}, T)+\frac{1}{\nu} \frac{\partial}{\partial c} W_{\text {mixing }}(c, T) \frac{\partial}{\partial \mathbf{F}} J
$$

in which solvent concentration $c$ can be eliminated from $W$ using the swelling constraint [28]. Now, if the free energy function is defined properly, the stress field for any boundary value problem will be calculated. In this work, considering the chain's inextensibility, the Gent model is employed to describe the network's elastic deformation which is expressed in terms of the deformation as follows $[26,34]$ :

$$
W_{\text {stretch }}=\frac{1}{2} N K T J_{m}\left(\log \left(1-\frac{I_{1}-3}{J_{m}}\right)-\frac{2 \log (J)}{J_{m}}\right)_{(9)}
$$

where $N$ and $K$ are density of the polymer chains and 
Boltzmann constant, respectively. In addition, $I_{1}$ is the first invariant of $\mathbf{C}$. The inextensibility of the network is implemented in the model by introducing locking parameter $J_{m}$ for the network, which is equal to the value of $\left(I_{1}-3\right)$ at the locking state of the network. When $\left(I_{1}-3\right)$ approaches the critical value of $J_{m}$, the strain energy starts to grow and prevents the huge deformation of the network. On the other hand, based on the Florry-Huggins theory, the mixing part of the free energy density is $[35,36]$ :

$$
\begin{aligned}
& W_{\text {mixing }}=K T\left(c \log \left(\frac{\nu c}{1+\nu c}\right)+\frac{\chi c}{1+\nu c}\right), \\
& \chi=\chi_{0}+\phi \chi_{1}, \quad \chi_{0}=A_{0}+B_{0} T, \\
& \chi_{1}=A_{1}+B_{1} T,
\end{aligned}
$$

where $\phi$ is the polymer volume fraction, respectively. Moreover, $\chi$ is the interaction parameter of the network that is a function of temperature and the polymer volume fraction for the temperature-sensitive PNIPAM hydrogel. In addition, $A_{0}, B_{0}, A_{1}$, and $B_{1}$ are material parameters adopted for the experiments reported by Afroze et al. [37]. Using a swelling constraint, Eq. (6), the total free energy density is:

$$
\begin{aligned}
W= & \frac{1}{2} N K T J_{m}\left(\log \left(1-\frac{I_{1}-3}{J_{m}}\right)-\frac{2 \log (J)}{J_{m}}\right) \\
& +\frac{K T}{\nu}(J-1)\left(\log \left(\frac{J-1}{J}\right)+\frac{\chi}{J}\right) .
\end{aligned}
$$

However, this statement of the free energy suffers from multiple solution and instability problem in the vicinity of the phase transition temperature. This problem was solved by Mazaheri et al. [28] through the modification of the free energy function and the snap-through instability; besides, the multiple-solution problem in

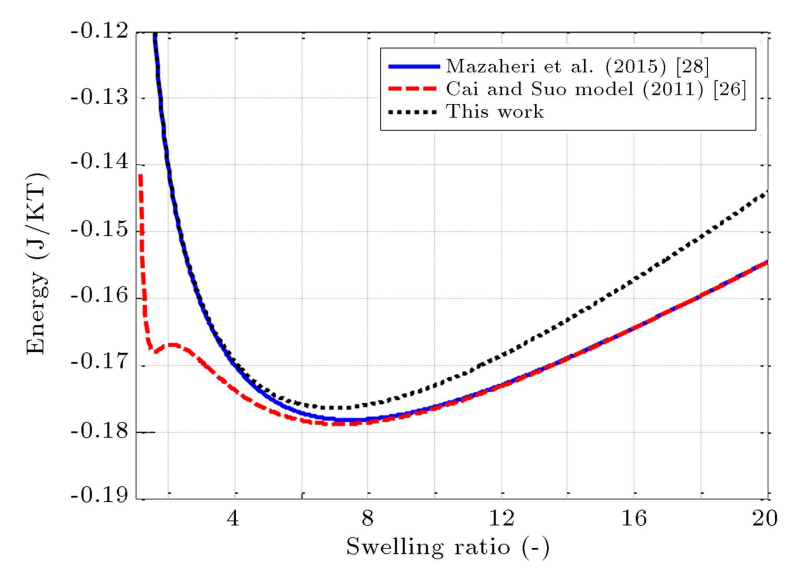

(a) the vicinity of PTT was eliminated for temperaturesensitive PNIPAM hydrogels. The modified free energy is:

$$
\begin{aligned}
W= & \frac{1}{2} N K T J_{m}\left(\log \left(1-\frac{I_{1}-3}{J_{m}}\right)-\frac{2 \log (J)}{J_{m}}\right) \\
& +\frac{K T}{\nu}(J-1)\left(-\frac{1}{J}-\frac{1}{2 J^{2}}-\frac{1}{3 J^{3}}+\frac{\chi}{J}\right) \\
= & \frac{1}{2} N K T J_{m}\left(\log \left(1-\frac{I_{1}-3}{J_{m}}\right)-\frac{2 \log (J)}{J_{m}}\right) \\
& +\frac{K T}{\nu}(J-1)\left(-\frac{1}{J}-\frac{1}{2 J^{2}}-\frac{1}{3 J^{3}}+\frac{\chi_{0}}{J}+\frac{\chi_{1}}{J^{2}}\right)
\end{aligned}
$$

In this equation, there exists one extra curve-fitting parameter $J_{m}$ which can result in better agreement of the model with the experimental data. As an example, for free swelling and equilibrium state, the free energy is calculated and shown herein for $T=305 \mathrm{~K}, N \nu=0.01$, and $J_{m}=100$. This temperature is close to phase transition temperature of the hydrogel where the model of Cai and Suo [26] experiences the multiple-solution problem solved by Mazaheri et al. [28] as depicted in Figure 1(a). The free swelling problem is solved for the present work model and shown in Figure 1(b). As shown in this figure, the present work has a continuous behavior in addition to the ability of considering chain inextensibility, especially for larger values of swelling ratio in which this work predicts a smaller value of swelling ratio in comparison with two other models. On the other hand, as is obvious in Figure 1(a) and (b), the present work has also continuous behavior; for larger values of the swelling ratio, the free energy value is larger than two other models. This means that the deformation of the network predicted through this work is smaller than two other models due to inextensibility

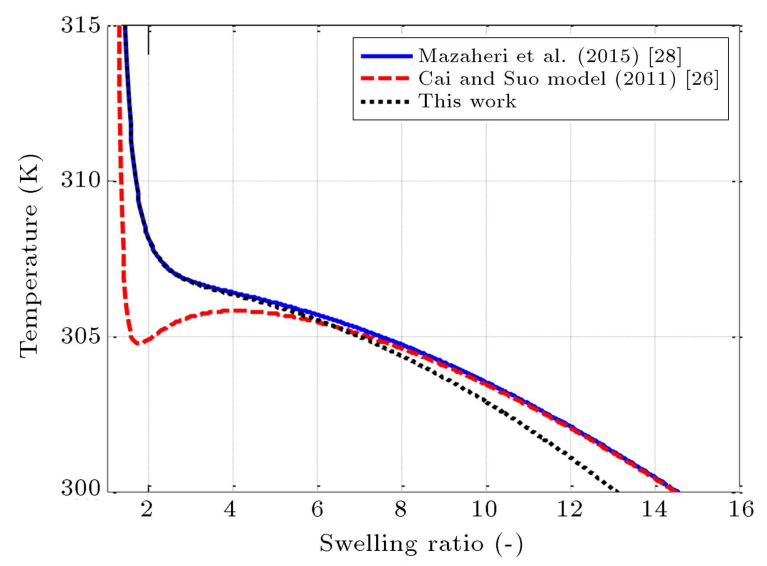

(b)

Figure 1. Comparison between this work and models of Mazaheri et al. [28] and Cai and Suo [26] for free swelling of a hydrogel with $N \nu=0.01$ and $J_{m}=100$ : (a) Free energy at $305.5 \mathrm{~K}$ and (b) free swelling problem due to temperature changes. 
of the network chains by introducing locking parameter $J_{m}$ in the present work.

As mentioned above, the present work can provide a better tool for predicting the experimental data due to introduction of extra parameter $J_{m}$. In the next section, using the present work model, first, the ability of the present work for the better prediction of experimental data is illustrated; thereafter, the model is implemented for solving some boundary value problems.

\section{Results and discussion}

In the previous section, the modified model is presented in detail. In this section, using the modified model, some homogeneous and inhomogeneous swelling problems are solved to show the validity of the model and the capability of the model for better prediction of the experimental data. In this regard, considering the experimental data available in the literature, free swelling and unidirectional swelling of the temperaturesensitive hydrogel are studied to validate the model in Section 3.1. Then, inhomogeneous swelling of the hydrogel is investigated for a spherical shell of PNIPAM on a hard core both analytically and numerically, in Section 3.2. Finally, in the same section, inhomogeneous swelling of a hydrogel bilayer is solved numerically.

\subsection{Homogeneous deformations}

\subsubsection{Free swelling}

As mentioned before, in addition to continuous behavior, the modified model has an extra locking parameter $J_{m}$ in comparison with models of Cai and Suo [26] and Mazaheri et al. [28], respectively. This extra parameter results in the best curve-fitting between the model results and the experimental data. With this fact in mind, the modified model is employed to investigate the free swelling problem and validate the model by experimental data available in the literature. For isotropic free swelling conditions, all stretches are equal and can be stated in terms of swelling ratio as follows:

$$
\lambda_{1}=\lambda_{2}=\lambda_{3}=\lambda=\left(\frac{V}{V_{0}}\right)^{1 / 3},
$$

where $\lambda_{i}$ and $V / V_{0}$ are principal stretches and swelling ratio, respectively. By equating the stress to zero, the swelling ratio at the equilibrium state is obtained versus temperature changes and shown in Figure 2 for the modified model, model of Mazaheri et al. [28], and model of Cai and Suo [26] besides the experimental data presented by $\mathrm{Oh}$ et al. [38] for free swelling problem.

As shown in Figure 2, both the modified model and model of Mazaheri et al. [28] have continuous
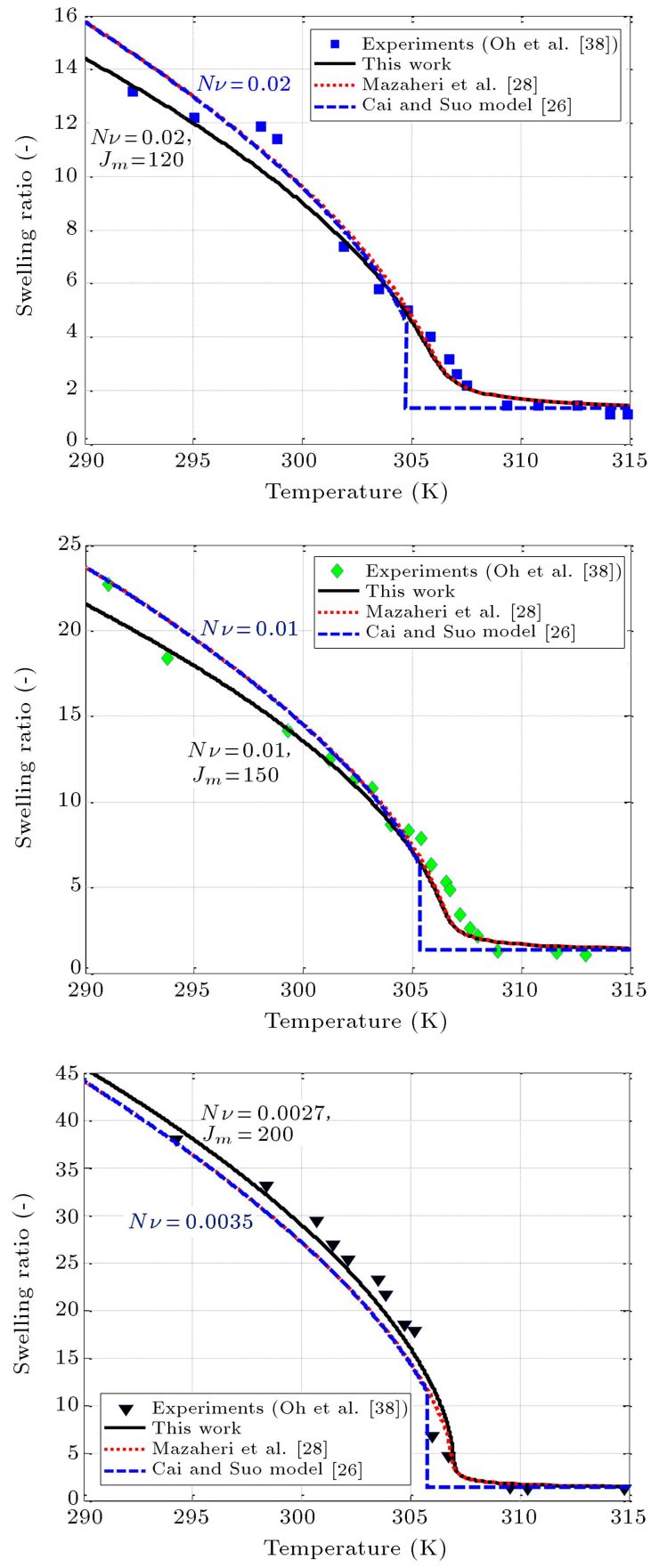

Figure 2. Comparison of experimental data and the modified model with models of Mazaheri et al. [28] and Cai and Suo [26] for free swelling. Experimental data are from the work of Oh et al. [38].

behavior in the vicinity of the PTT, while the model of Cai and Suo [26] shows discontinuity in this region. In addition to the continuity in the vicinity of the PTT, the modified model shows better agreement with the experimental data, especially with respect to the larger amount of the swelling ratio values due to the introduction of the locking parameter in the modified model. Adding extra curve-fitting parameter $J_{m}$, 
which models a physical phenomenon that originates from inextensibility of the network chains, results in a better prediction of the experimental data. Thus, the modified model can predict the hydrogel behavior with better agreement and with the capability of continuous behavior prediction, particularly with respect to larger values of swelling ratio and smaller values of $N \nu$, which is a measure of cross-linking density.

\subsubsection{Unidirectional constrained swelling}

The next problem considered in this section is the unidirectional constrained swelling of the network in which the stretch in the constraint direction is fixed and equal to $\lambda_{1}$, while the other two stretches vary with the temperature changes $\left(\lambda_{2}=\lambda_{3}\right)$. Using the experimental data reported by Suzuki et al. [39], free swelling of a cubic piece of the hydrogel at $303 \mathrm{~K}$ with equilibrium stretch $\lambda_{0}$ is investigated. Thereafter, the cube can be constrained in one direction with a fixed stretch equal to $\lambda_{1}=n \lambda_{0}(n=1,2,3)$ and, in the other two directions, the cube is free and experiences deformation with the temperature changes [28]. By substituting the longitudinal stretch in the model and minimizing the free energy, the equilibrium state is identified for different values of $\lambda_{1}$, and the calculated results are shown in Figure 3 for the modified model, model of Mazaheri et al. [28], and model of Cai and Suo [26] besides the experimental data presented by Suzuki et al. [39]. As depicted in Figure 3, similar to free swelling problem, the modified model is in good agreement with the experimental data, especially with respect to larger values of swelling ratio and smaller values of $\lambda_{1}$ due to larger values of deformation, which activates locking phenomenon in the network. On the other hand, as expected, the modified model shows a continuous behavior in the vicinity of PTT both

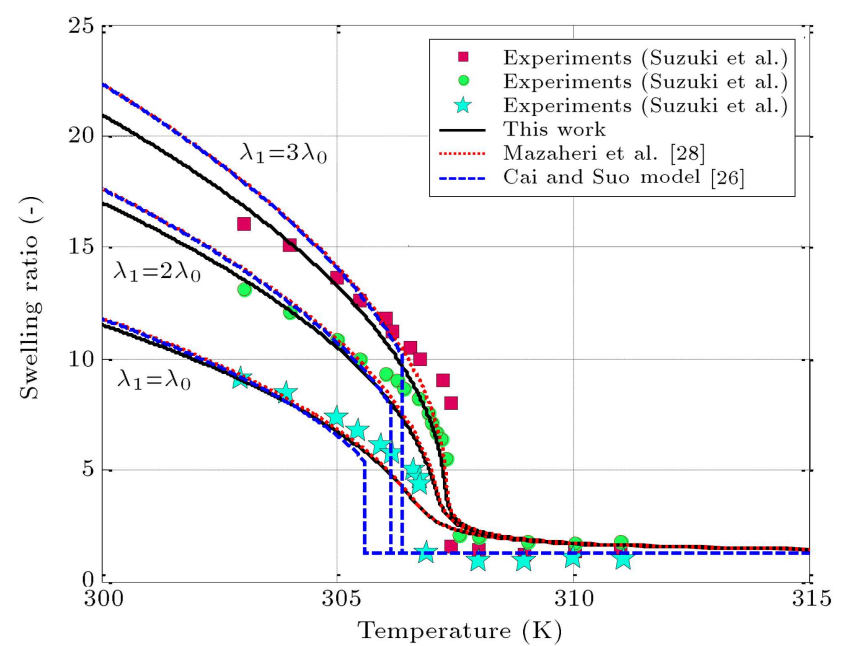

Figure 3. Comparison of experimental data and the modified model with models of Mazaheri et al. [28] model and Cai and Suo [26] for unidirectional swelling. for free and unidirectional constraint swellings of the hydrogel and can be implemented in inhomogeneous swelling problems without encountering any instability and discontinuity [28]. In addition, taking the chain locking into account leads to better curve fitting which makes the modified model appropriate for a better prediction of experimental data.

\subsection{Inhomogeneous deformations}

Due to the existence of inhomogeneous deformations in many practical applications of the hydrogels, in this section, the inhomogeneous swelling of the network due to temperature changes is studied and, the effect of inextensibility of the networks chains is investigated. First, inhomogeneous swelling of a spherical hydrogel shell on a hard core is considered both analytically and numerically. Then, using analytical results, the numerical scheme is validated; finally, inhomogeneous deformation of a bilayer beam is numerically studied, and the chains' inextensibility effect is distinguished.

\subsubsection{Swelling of a spherical shell on a hard core}

The schematic draw of the spherical shell on a hard core is shown in Figure 4. The shell is assumed to be attached to the hard core at a preparation temperature which is equal to $310 \mathrm{~K}$, and all changes are calculated with respect to this free-stress state (reference state) in which the shell experiences an isotropic stretch of $\lambda_{0}=1.18$ for $N \nu=0.01$ and $J_{m}=50$. Thus, $I_{1}$ and $J$ in the free energy statement should be modified by multiplying $\lambda_{0}^{2}$ by $\lambda_{0}^{3}$, respectively, and the free energy should be divided by $\lambda_{0}^{3}$. Moreover, $R_{i}$ and $R_{o}$ are inner and outer radii of the spherical shell in the reference state, respectively; $R$ is radius of an arbitrary element in the reference state.

As shown in Figure 4, due to the symmetry, all field variables are functions of $R$, and the equilibrium equation in the radial direction gives:

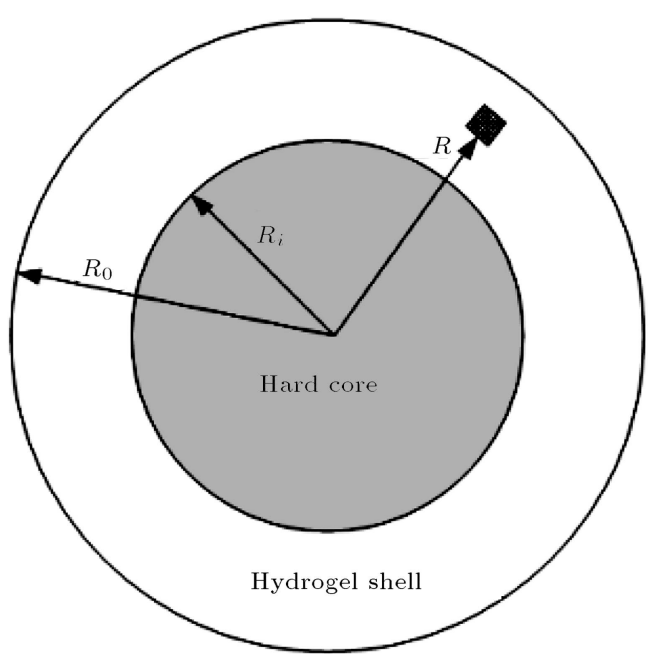

Figure 4. Schematic of the spherical hydrogel shell coated on a hard core. 


$$
\frac{d P_{r}}{d R}-\frac{2\left(P_{r}-P_{\theta}\right)}{R}=0
$$

where $P_{r}$ and $P_{\theta}$ are the nominal radial and hoop stresses, respectively. In addition, by defining $r$ as the radius of an element of the hydrogel in the current state, the stretch components are obtained as follows:

$$
\lambda_{r}=\frac{r}{R}, \quad \lambda_{\theta}=\frac{d}{d R} r(R) .
$$

As a result, by substituting stretches in stress and, subsequently, substituting stresses in equilibrium equation, a differential equation in term of $r(R)$ is obtained, which can be solved by introducing the relevant boundary conditions and using a finite difference technique with Richardson extrapolation [40]. The boundary conditions are:

$$
r=\bar{\lambda} R_{i} \quad \text { at } \quad R=R_{i}, \quad P_{r}=0 \quad \text { at } \quad R=R_{o},
$$

where $\bar{\lambda}$ is the initial stretch in the inner radius and is assumed to be 1.001 [41]. The governing boundary value problem is solved for a shell with $R_{o} / R_{i}=2$ for the modified model, and model of Mazaheri et al. [28] and their results (marked by ANL) are depicted in Figure 5 for normalized displacement of the outer radius of the shell (normalized by its value at reference state). As shown in this figure, a decrease of temperature from the reference state leads to an increase in the outer radius of the shell; however, this increase is smaller in the results of this work due to considering chain locking of the network. The obtained results show the importance of considering this parameter in practical problems. On the other hand, both the models predict large variations in the vicinity of PTT, which is in agreement with the temperature-sensitive PNIPAM hydrogels behavior studied in the homogeneous swelling problems (studied in the previous section).

To provide a powerful tool for solving complicated coupled thermo-mechanical problems, one should implement the modified model in a numerical finiteelement framework. Thus, in the next step, the modified model is implemented in commercial software of ABAQUS by writing a UHYPER subroutine for both the modified model and the model of Mazaheri et al. [28] by which the problem of swelling of a spherical shell solved in this section is resolved, and the results (marked as FEM) are shown in Figure 5 again. As seen, the FEM results are in very good agreement with analytical ones, implying the validity of numerical method for the modified model, and the prepared UHYPER subroutine can be used in other problems.

\subsubsection{Inhomogeneous swelling of a temperature-sensitive bilayer}

As another applied problem, the inhomogeneous swelling of a bilayer composed of an active

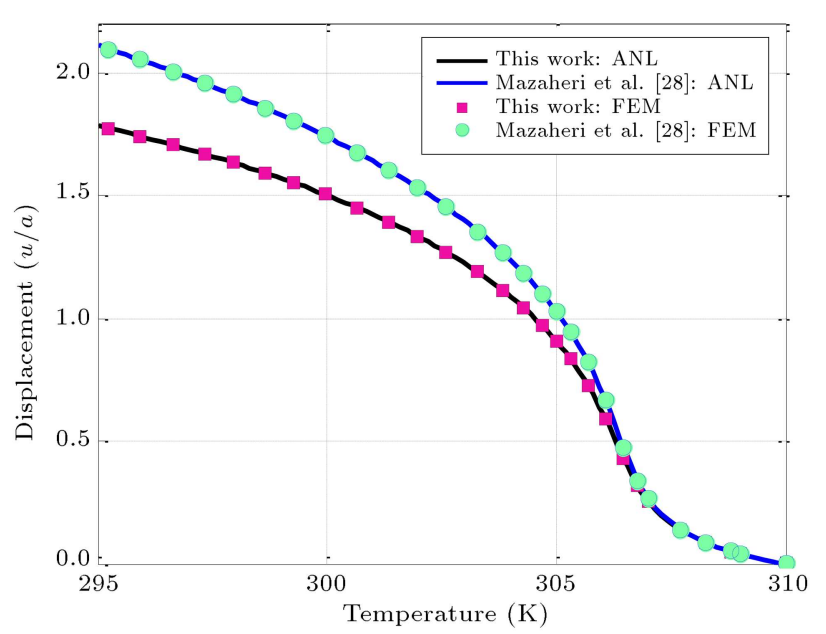

Figure 5. Analytical (ANL) and finite-element (FEM) results for displacement of outer radius of spherical shell obtained using this work model and model of Mazaheri et al. [28].

temperature-sensitive hydrogel layer resting on a neutral elastomer layer is considered. The hydrogel layer is bound to the sub-layer at a reference temperature equal to $310 \mathrm{~K}$ with a stress-free condition. Temperature decrease brings about a swelling of the hydrogel layer; as a result, the bilayer is bent and deformed that can be used as a sensor and an actuator. The neutral layer behavior is modelled by a neo-Hookean model with a shear modulus of $G$ where $G=0.0001 \frac{N K T}{2}$. Through the modified model and model of Mazaheri et al. [28], bending the bilayer is simulated due to temperature changes, and the results are shown in Figure 6(a) and (b), respectively.

As shown in Figure 6, in comparison with the model of Mazaheri et al. [28], the chains' inextensibility in this work results in smaller displacement in the same temperatures. This phenomenon is important and should be considered in the design process of such actuators. For a better comparison, curvature of the beam and vertical displacement of a point in the midspan of the beam are calculated and shown in Figure 7. The obtained results show the difference between this work and the work of Mazaheri et al. [28] due to the locking parameter introduction in the modified model, which can be crucial in applied problems.

Thus, the results obtained in this section for the inhomogeneous swelling problems show the ability of the modified model to study these problems without encountering any instability besides the consideration of the locking phenomenon in the network swelling. In addition, the numerical tool can be used for solving any boundary value problem in the applied fields.

\section{Summery and conclusions}

In this work, the equilibrium behavior of the 

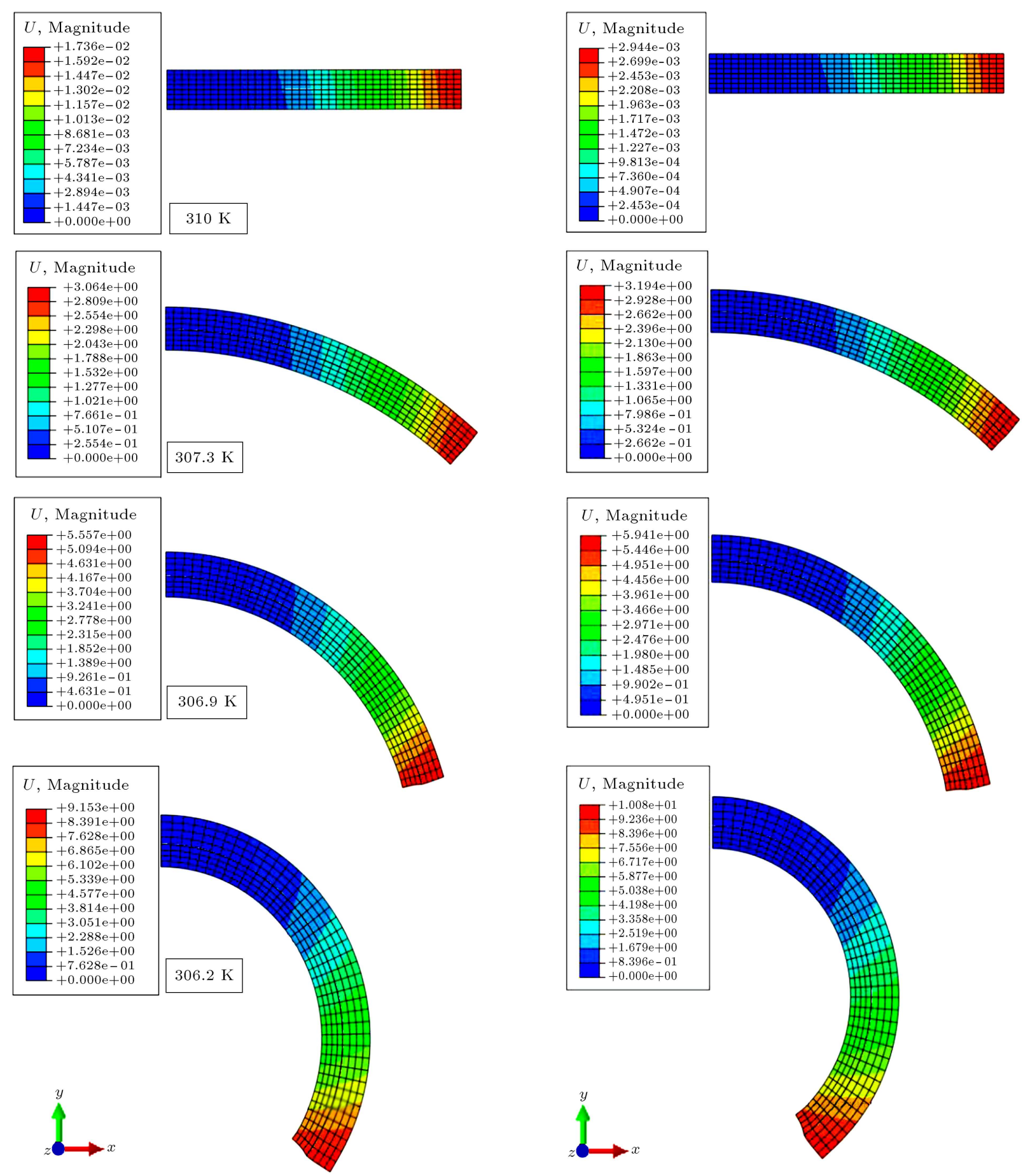

(a)

(b)

Figure 6. Comparison of this work (a) with results of Mazaheri et al. [28] and (b) for inhomogeneous swelling of a bilayer beam made of the temperature-sensitive hydrogel with $N \nu=0.01$ and $J_{m}=50$.

temperature-sensitive hydrogels was considered with an emphasis on the locking phenomenon in the polymer chains in the large deformation regime. In this regard, by assuming the additive decomposition of the free energy, first, the Gent model was used to describe the elastic part of the free energy, which inherently considers the locking phenomenon. On the other hand, the mixing part of the free energy was modified to eliminate instability and multiple-solution problems. Thereafter, the modified model was implemented in some homogeneous and inhomogeneous problems. Firstly, the free and unidirectional constraint swelling problems were studied, and the obtained results were compared with experimental data, showing good agreement, especially 

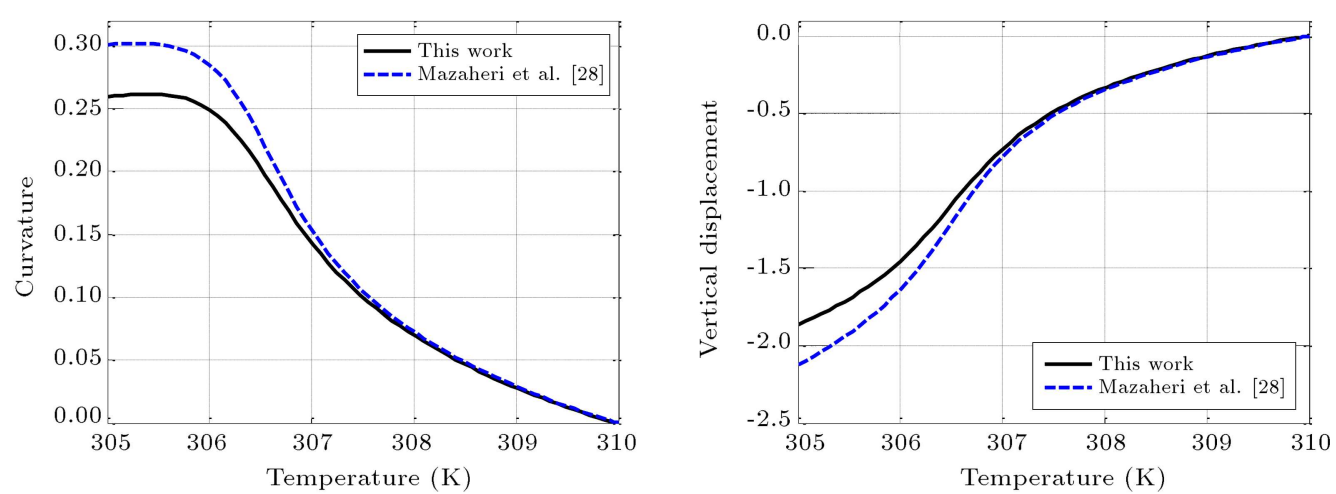

Figure 7. Comparison of this work with results of Mazaheri et al. [28] for vertical displacement and curvature of swelling of a bilayer beam made of the temperature-sensitive hydrogel with $N \nu=0.01$ and $J_{m}=50$.

with respect to larger values of swelling ratio. In addition, introduction of locking parameter, $J_{m}$, in the model resulted in better curve fitting between the modified model and the experimental data for smaller values of cross-linking density of the network and larger values of the swelling ratio. Thus, the modified model was in better agreement with the experimental data due to the consideration of the physical phenomenon of chain locking in the network and had a good potential for use in applied problems.

The continuous behavior of the model makes it appropriate for inhomogeneous problems and numerical methods. Thus, in the next step, the modified model was employed to solve the inhomogeneous swelling of a hydrogel spherical shell attached to a hard core both analytically and numerically. The numerical methods were developed in ABAQUS software by writing a user subroutine, and the obtained results were in very good agreement with analytical ones that guaranteed viable performance of the numerical method. Thenceforth, the inhomogeneous swelling of a bilayer beam due to temperature changes was solved, and the results were presented. The obtained results showed the importance of considering chain locking in the temperature-sensitive hydrogels, especially with respect to smaller values of cross-linking density and larger values of swelling ratio. Thus, the main advantage of the modified model is continuity besides its ability to consider the network chain locking.

\section{References}

1. Depa, K., Strachota, A., Šlouf, M., and Hromádková, J. "Fast temperature-responsive nanocomposite PNIPAM hydrogels with controlled pore wall thickness: Force and rate of T-response", European Polymer Journal, 48(12), pp. 1997-2007 (2012).

2. Chang, D.P., Dolbow, J.E., and Zauscher, S. "Switchable friction of stimulus-responsive hydrogels", Langmuir, 23(1), pp. 250-257 (2007).

3. Wang, J., Chen, Z., Mauk, M., et al. "Self-actuated, thermo-responsive hydrogel valves for lab on a chip", Biomedical Microdevices, 7(4), pp. 313-322 (2005).

4. Marcombe, R., Cai, S., and Hong, W. "A theory of constrained swelling of a pH-sensitive hydrogel", Soft Matter, 6, pp. 784-793 (2010).

5. Kurnia, J.C., Birgersson, E., and Mujumdar, A.S. "Analysis of a model for pH-sensitive hydrogels", Polymer, 53(2), pp. 613-622 (2012).

6. Mazaheri, H., Baghani, M., Naghdabadi, R., and Sohrabpour, S. "Coupling behavior of the $\mathrm{pH} /$ temperature sensitive hydrogels for the inhomogeneous and homogeneous swelling", Smart Materials and Structures, 25(8), p. 085034 (2016).

7. Chester, S.A. and Anand, L. "A coupled theory of fluid permeation and large deformations for elastomeric materials", Journal of the Mechanics and Physics of Solids, 58(11), pp. 1879-1906 (2010).

8. Doi, M. "Gel dynamics", Journal of the Physical Society of Japan, 78(5), p. 052001 (2009).

9. Hong, W., Zhao, X., and Suo, Z. "Large deformation and electrochemistry of polyelectrolyte gels", Journal of the Mechanics and Physics of Solids, 58(4), pp. 558577 (2010).

10. Li, J., Suo, Z., and Vlassak, J.J. "A model of ideal elastomeric gels for polyelectrolyte gels", Soft Matter, 10(15), pp. 2582-2590 (2014).

11. Ahn, S.K., Kasi, R.M., Kim, S.C., et al. "Stimuliresponsive polymer gels", Soft Matter, 4(6), pp. 11511157 (2008).

12. Zrínyi, M., Szilágyi, A., Filipcsei, G., et al. "Smart gel-glass based on the responsive properties of polymer gels", Polymers for Advanced Technologies, 12(9), pp. 501-505 (2001).

13. Toh, W., Ng, T.Y., Hu, J., and Liu, Z. "Mechanics of inhomogeneous large deformation of photo-thermal sensitive hydrogels", International Journal of Solids and Structures, 51(25-26), pp. 4440-4451 (2014).

14. Osada, Y. and Gong, J.P. "Soft and wet materials: polymer gels", Advanced Materials, 10(11), pp. 827837 (1998). 
15. Li, H. "Kinetics of smart hydrogels responding to electric field: A transient deformation analysis", International Journal of Solids and Structures, 46(6), pp. 1326-1333 (2009).

16. Hoffman, A.S. "Hydrogels for biomedical applications", Advanced Drug Delivery Reviews, 54(1), pp. 3-12 (2002).

17. Randhawa, J.S., Laflin, K.E., Seelam, N., and Gracias, D.H. "Microchemomechanical systems", $A d$ vanced Functional Materials, 21(13), pp. 2395-2410 (2011).

18. Abdolahi, J., Baghani, M., Arbabi, N., and Mazaheri, $\mathrm{H}$. "Analytical and numerical analysis of swellinginduced large bending of thermally-activated hydrogel bilayers", International Journal of Solids and Structures, 99, pp. 1-11 (2016).

19. Richter, A., Klatt, S., Paschew, G., and Klenke, C. "Micropumps operated by swelling and shrinking of temperature-sensitive hydrogels", Lab on a Chip, 9(4), pp. 613-618 (2009).

20. Xia, C., Lee, H., and Fang, N. "Solvent-driven polymeric micro beam Device", Journal of Micromechanics and Microengineering, 20(8), p. 085030 (2010).

21. Westbrook, K.K. and Qi, H.J. "Actuator designs using environmentally responsive hydrogels", Journal of Intelligent Material Systems and Structures, 19, pp. 597-607 (2008).

22. Eddington, D.T. and Beebe, D.J. "Flow control with hydrogels", Advanced Drug Delivery Reviews, 56(2), pp. 199-210 (2004).

23. Mazaheri, H., Baghani, M., Naghdabadi, R., and Sohrabpour, S. "Inhomogeneous swelling behavior of temperature sensitive PNIPAM hydrogels in microvalves: analytical and numerical study", Smart $M a$ terials and Structures, 24(4), p. 045004 (2015).

24. Arbabi, N., Baghani, M., Abdolahi, J., et al. "Study on pH-sensitive hydrogel micro-valves: A fluid-structure interaction approach", Journal of Intelligent Material Systems and Structures, 28(12), pp. 1589-1602 (2016).

25. Chester, S.A. and Anand, L. "A thermo-mechanically coupled theory for fluid permeation in elastomeric materials: Application to thermally responsive gels", Journal of the Mechanics and Physics of Solids, 59(10), pp. 1978-2006 (2011).

26. Cai, S. and Suo, Z. "Mechanics and chemical thermodynamics of phase transition in temperature-sensitive hydrogels", Journal of the Mechanics and Physics of Solids, 59(11), pp. 2259-2278 (2011).

27. Birgersson, E., Li, H., and Wu, S. "Transient analysis of temperature-sensitive neutral hydrogels", Journal of the Mechanics and Physics of Solids, 56(2), pp. 444466 (2008).
28. Mazaheri, H., Baghani, M., and Naghdabadi, R. "Inhomogeneous and homogeneous swelling behavior of temperature-sensitive poly-( $\mathrm{N}$-isopropylacrylamide) hydrogels", Journal of Intelligent Material Systems and Structures, 27(3), pp. 324-336 (2016).

29. Ji, H., Mourad, H., Fried, E., and Dolbow, J. "Kinetics of thermally induced swelling of hydrogels", International Journal of Solids and Structures, 43(7-8), pp. 1878-1907 (2006).

30. Hong, W., Zhao, X., Zhou, J., and Suo, Z. "A theory of coupled diffusion and large deformation in polymeric gels", Journal of the Mechanics and Physics of Solids, 56(5), pp. 1779-1793 (2008).

31. Hong, W., Liu, Z., and Suo, Z. "Inhomogeneous swelling of a gel in equilibrium with a solvent and mechanical load", International Journal of Solids and Structures, 46(17), pp. 3282-3289 (2009).

32. Li, J., Hu, Y., Vlassak, J.J., and Suo, Z. "Experimental determination of equations of state for ideal elastomeric gels", Soft Matter, 8(31), pp. 8121-8128 (2012).

33. Cai, S. and Suo, Z. "Equations of state for ideal elastomeric gels ", EPL (Europhysics Letters), 97(3), p. 34009 (2012).

34. Patra, K. and Sahu, R.K. "A visco-hyperelastic approach to modelling rate-dependent large deformation of a dielectric acrylic elastomer", International Journal of Mechanics and Materials in Design, 11(1), pp. 79-90 (2015).

35. Flory, P.J. and Rehner Jr, J. "Statistical mechanics of cross-linked polymer networks II. Swelling", The Journal of Chemical Physics, 11(11), pp. 521-526 (1943).

36. Huggins, M.L. "Some properties of solutions of longchain compounds", Journal of Physical Chemistry, 46(1), pp. 151-158 (1942).

37. Afroze, F., Nies, E., and Berghmans, H. "Phase transitions in the system poly(N-isopropylacrylamide)/water and swelling behaviour of the corresponding networks", Journal of Molecular Structure, 554(1), pp. $55-68$ (2000).

38. Oh, K.S., Oh, J.S., Choi, H.S., and Bae, Y.C. "Effect of cross-linking density on swelling behavior of NIPA gel particles", Macromolecules, 31(21), pp. 7328-7335 (1998).

39. Suzuki, A., Sanda, K., and Omori, Y. "Phase transition in strongly stretched polymer gels", Journal of Chemical Physics, 107(13), pp. 5179-5185 (1997).

40. Ascher, U.M., Mattheij, R.M.M., and Russell, R.D., Numerical Solution of Boundary Value Problems for Ordinary Differential Equations, 478, Prentice Hall New Jersey (1988).

41. Zhao, X., Hong, W., and Suo, Z. "Inhomogeneous and anisotropic equilibrium state of a swollen hydrogel containing a hard core", Applied Physics Letters, 92(5), p. 051904 (2008). 


\section{Biography}

Hashem Mazaheri received his BS, MS, and PhD degrees in Mechanical Engineering from Sharif University of Technology, Tehran, Iran in 2007, 2010, and 2015, respectively. He is now an Assistant Profes- sor at the Mechanical Engineering Department, BuAli Sina University, Hamedan, Iran. His research interests include solid mechanics, computational mechanics, nonlinear finite-element method, constitutive modelling of materials especially smart hydrogels and design problems. 